\title{
Increased Uptake of Voluntary Medical Male Circumcision (VMMC) Services among Older Men Following Mobile Technology Demand Creation in Shinyanga and Simiyu, Tanzania
}

\author{
Godfrey J. Kundi', Lucy Mphuru', James McMahan'1, Paul Mwakipesile', Salli Mwanasalli², \\ Daimon Simbeye ${ }^{2}$, Peter Masika ${ }^{3}$ and Lorie L. Broomhall ${ }^{\star 1}$
}

${ }^{1}$ Monitoring, Evaluation and Research, Intrahealth International, Chapel Hill, NC, USA; ${ }^{2}$ Centers for Disease Control, Atlanta, GA, USA; ${ }^{3}$ 4. Tanzania Youth Alliance (TAYOA), Dar es Salaam, United Republic of Tanzania

\section{Objective}

To describe the success of mobile text messaging and community sensitization activities to increase the number of men 20 years and above accessing VMMC services in Tanzania.

\section{Introduction}

In 2007, the United Nations recommended provision of VMMC in settings with high HIV prevalence and low rates of male circumcision (MC). This recommendation was based on randomized studies in Africa, which demonstrated that MMC reduces HIV acquisition by $38 \%-66 \%$ [1]. Tanzania was among 13 countries selected for early implementation. In response, IntraHealth International, through the US President's Emergency Plan for AIDS Relief (PEPFAR) and Centers for Disease Control and Prevention (CDC) supported the Ministry of Health and Social Welfare (MOHSW) to launch a VMMC program in Shinyanga and Simiyu regions where HIV prevalence is high (7.4\%) and VMMC is low (30-32\%). Older men age 30-49 have the highest risk of contracting HIV and, thus, are in greater need of the protective effects of VMMC; however, significant cultural and attitudinal barriers hinder $\mathrm{MC}$ acceptance and uptake in this population. For example, from Oct 2010-Sep 2012, 65,812 males in Tanzania were circumcised, yet only $7.1 \%$ were men over 20 years. To maximize impact, the project implemented an intensive social mobilization initiative using mobile text messaging (SMS) and community sensitization activities targeting men over 20 years.

\section{Methods}

From October 2012, IntraHealth partnered with the Tanzania Youth Alliance (TAYOA) to conduct a demand creation intervention for older men using mobile texting technology. IntraHealth developed messages targeting men 20 years and above using the national VMMC information booklet. In collaboration with district councils, IntraHealth and TAYOA organized community meetings with strategic VMMC messages to sensitize community members to the availability and benefits of VMMC. TAYOA mobilizers recruited clients during community sensitization activities two weeks prior to VMMC outreach campaigns. Clients who expressed interest in VMMC filled out a subscription card including their mobile number, place of residence, and consent to receive free SMS messages. The client information was entered into the TAYOA software. Enrolled clients subsequently received SMS messages with information on the health benefits of VMMC for men and women, services provided, and availability and dates of VMMC services. SMS reminders were sent to clients about service locations and times a week before each VMMC outreach campaign. The clients who eventually obtained VMMC services filled in another card at the facility and received post-circumcision and follow-up instructions on proper wound care via SMS.

\section{Results}

Before the demand creation intervention, only $7.1 \%$ of men circumcised were 20 years and above during the two-year period from Oct 2010-Sep 2012. During the nine-month intervention period (Oct 2012-Jun 2013), a total of 63,873 men and boys were circumcised. In quarter one of the project, $22 \%(5,054)$ were 20 years or older; this proportion rose to $28 \%(5,364)$ in quarter two and $32 \%(6,968)$ in quarter three for an overall increase of $25 \%$. As further evidence of the intervention's effectiveness, during the third quarter, $29 \%(6,382)$ of clients were referred to the VMMC services by TAYOA SMS.

\section{Conclusions}

The data show an increase in VMMC uptake by men 20 years and older following community sensitization and demand creation using mobile technology. Self-reported SMS referrals suggest that these techniques are highly effective at reaching the target group. The project will continue to use text message technology and explore other methods to increase VMMC demand by men 20 years and older.

\section{Keywords}

circumcision; Tanzania; older men; demand creation

\section{References}

(1) Siegfried N, Muller M, Deeks JJ, Volmink J. Male circumcision for prevention of heterosexual acquisition of HIV in men. Cochrane Database of Systematic Reviews 2009, Issue 2. Art. No. CD003362. DOI: 10.1002/14651858.CD003362.pub2.

(2) PEPFAR's best practices for voluntary medical male circumcision site operations: A service guide for site operations. PEPFAR, 2012.

*Lorie L. Broomhall

E-mail: Ibroomhall@intrahealth.org 